\title{
A Close Look at Charge Generation in Polymer:Fullerene Blends with Microstructure Control
}

\author{
Mariateresa Scarongella, ${ }^{\dagger}$ Jelissa De Jonghe-Risse, ${ }^{\dagger}$ Ester Buchaca-Domingo, ${ }^{\ddagger}$ Martina Causa, $\$$
}

Zhuping Fei," Martin Heeney, "Jacques-E. Moser, ${ }^{\dagger}$ Natalie Stingelin, ${ }^{\ddagger}$ and Natalie Banerji*, ${ }^{\dagger}$,

${ }^{\dagger}$ Institute of Chemical Sciences \& Engineering, Ecole Polytechnique Fédérale de Lausanne (EPFL), SB ISIC GR-MO, Station 6, CH-1015 Lausanne, Switzerland

${ }^{\ddagger}$ Centre for Plastic Electronics and Department of Materials, Imperial College London, Exhibition Road, London, SW7 2AZ, United Kingdom

${ }^{\S}$ Department of Chemistry, University of Fribourg, Chemin du Musée 9, CH-1700 Fribourg, Switzerland

${ }^{\|}$Centre for Plastic Electronics and Department of Chemistry, Imperial College London, Exhibition Road, London, SW7 2AZ, United Kingdom

\section{Supporting Information}

ABSTRACT: We reveal some of the key mechanisms during charge generation in polymer:fullerene blends exploiting our well-defined understanding of the microstructures obtained in pBTTT:PCBM systems via processing with fatty acid methyl ester additives. Based on ultrafast transient absorption, electroabsorption, and fluorescence up-conversion spectroscopy, we find that exciton diffusion through relatively phase-pure polymer or fullerene domains limits the rate of electron and hole transfer, while prompt charge separation occurs in regions

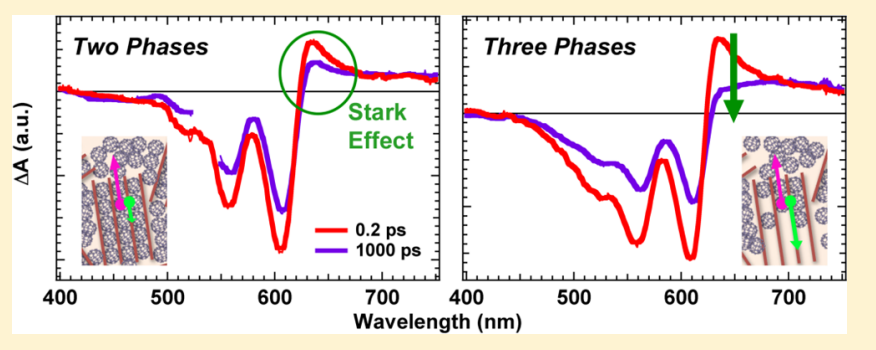
where the polymer and fullerene are molecularly intermixed (such as the co-crystal phase where fullerenes intercalate between polymer chains in PBTTT:PCBM). We moreover confirm the importance of neat domains, which are essential to prevent geminate recombination of bound electron-hole pairs. Most interestingly, using an electro-absorption (Stark effect) signature, we directly visualize the migration of holes from intermixed to neat regions, which occurs on the subpicosecond time scale. This ultrafast transport is likely sustained by high local mobility (possibly along chains extending from the co-crystal phase to neat regions) and by an energy cascade driving the holes toward the neat domains.

\section{INTRODUCTION}

Organic photovoltaic (OPV) cells offer a cost-efficient way to convert solar energy to electricity with over $10 \%$ efficiency. ${ }^{1,2}$ The photoactive material of such devices commonly consists of a conjugated polymer electron donor blended with an electronaccepting fullerene derivative, yielding an interpenetrating network known as the bulk heterojunction $(\mathrm{BHJ}){ }^{3,4}$ The excitons generated by light absorption in such BHJs are dissociated via charge separation (CS) between the polymer and fullerene, and the charges are transported through segregated pathways of the $\mathrm{BHJ}$ to opposite electrodes of the device.

Early on, it has become clear that the precise arrangement of the donor and acceptor in the $\mathrm{BHJ}$ (from the molecular to the microscopic scale), which we will refer to as microstructure, has strong implications on solar cell efficiency. ${ }^{5}$ Numerous empirical strategies to enhance device performance by influencing the microstructure of $\mathrm{BHJ}$ thin films during or after their solution-processing have been explored (e.g., casting solvent optimization, use of additives, thermal/solvent annealing). ${ }^{6-12}$ It is, however, only with recent advances in structural characterization that the true complexity of the $\mathrm{BHJ}$ has been revealed. ${ }^{13,14}$ Not only can the polymer and fullerene arrange into either amorphous or crystalline neat domains of variable size (i.e., relatively phase-pure regions), but an additional, intimately mixed polymer-fullerene phase has been identified. ${ }^{15-20}$ This can play an important role in the photovoltaic processes, and indeed, the highest device performance in polymer:fullerene blends is typically obtained with a three-phase microstructure consisting of the neat domains as well as intermixed regions. ${ }^{19-22}$ The better understanding of the $\mathrm{BHJ}$ provides opportunities to establish clear structureproperty relations that will enable precise control of the optoelectronic properties in OPV materials by targeting optimized microstructures. $^{22-29}$

In an effort to comprehensively correlate the early charge generation events to $\mathrm{BHJ}$ structure, we used ultrafast absorption and emission spectroscopy to investigate blends of poly(2,5-bis(3-hexadecylthiophen-2-yl)thieno [3,2-b] thiophene

Received: September 29, 2014

Published: February 4, 2015 
(pBTTT, inset of Figure 1A) and [6,6]-phenyl $\mathrm{C}_{60}$ butyric acid methyl ester (PCBM). Although pBTTT is mainly known for

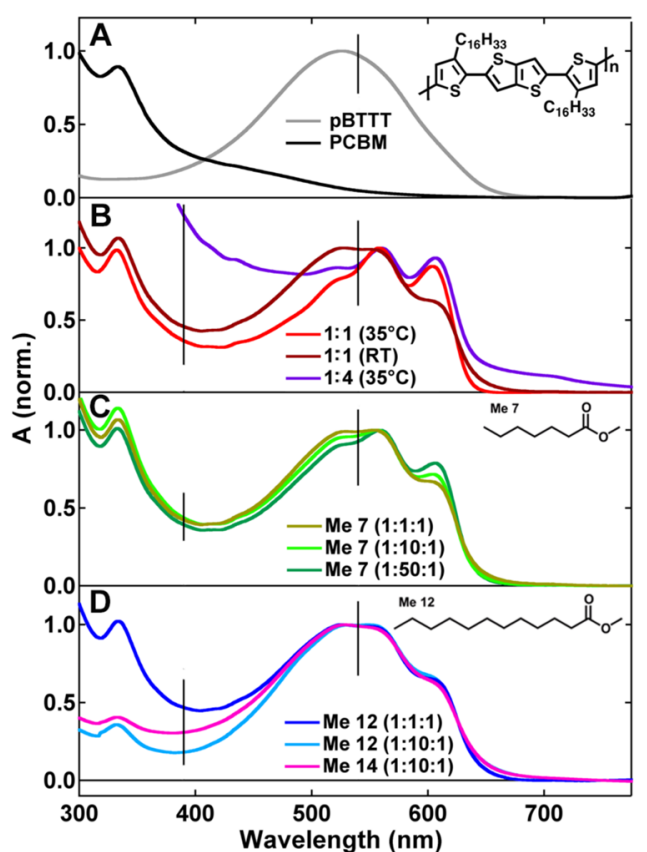

Figure 1. Steady-state absorption spectra of the investigated pBTTT, PCBM, and pBTTT:PCBM systems. (A) Neat films of pBTTT (molecular structure in inset) and PCBM. (B) pBTTT:PCBM blends in $1: 1$ and 1:4 ratio (by weight), wire bar coated at different temperatures. (C) 1:1 blends of pBTTT:PCBM with different amounts of processing additive Me 7 (structure shown in inset). (D) 1:1 blends of pBTTT:PCBM with different amounts of processing additive Me 12 (structure shown in inset) and Me 14. Vertical lines are the excitation wavelengths used for transient absorption spectroscopy.

excellent performance in field effect transistors, ${ }^{30-32}$ this material is also a particularly suitable model system to study microstructural effects in solar cells. It is known that PCBM molecules intercalate between the side chains of ordered polymer domains, forming a co-crystalline phase and providing a structurally well-defined setting for our investigation. ${ }^{18,20,22}$ Moreover, we have recently shown that the co-crystal formation can be controlled by using fatty acid methyl esters as additives, expanding the range of phase morphologies accessible with the polymer:fullerene blend from a fully intermixed, to a partially intercalated, to a predominantly nonintercalated microstructure. $^{24}$

We exploit these well-defined microstructures to focus on two main questions. First, we investigate how CS by both electron and hole transfer depends on microstructure, and show how its rate can be limited by exciton diffusion through neat domains. ${ }^{33-35}$ Second, we discuss the role played by the $\mathrm{BHJ}$ structure in the generation of free (as opposed to bound) charges, using the early evolution of an electro-absorption signature (Stark effect) in the transient absorption (TA) data. Indeed, there has been much debate about the time scale (ultrafast or slow) and mechanism by which the electron-hole pairs generated across polymer:fullerene interfaces separate to a distance at which they can overcome their mutual Coulomb attraction (for example, by long-range CS, via hot states, assisted by delocalization, by diffusive dissociation, or mediated by high local mobility). ${ }^{34,36-47}$

\section{RESULTS AND DISCUSSION}

2.1. Steady-State Absorption Spectra. The absorption spectrum of neat pBTTT shows a structureless band around $525 \mathrm{~nm}$, while neat PCBM films absorb little in the visible range, but mainly below $400 \mathrm{~nm}$ (Figure 1A). In the 1:1 pBTTT:PCBM blend (by weight), processed by wire bar coating at $35{ }^{\circ} \mathrm{C}$ (see the Supporting Information for details), there is quasi-complete intercalation of fullerenes between the polymer side chains, yielding an intimately intermixed cocrystal phase, as schematically shown in Figure $2 \mathrm{~A}^{20,22,24,48}$

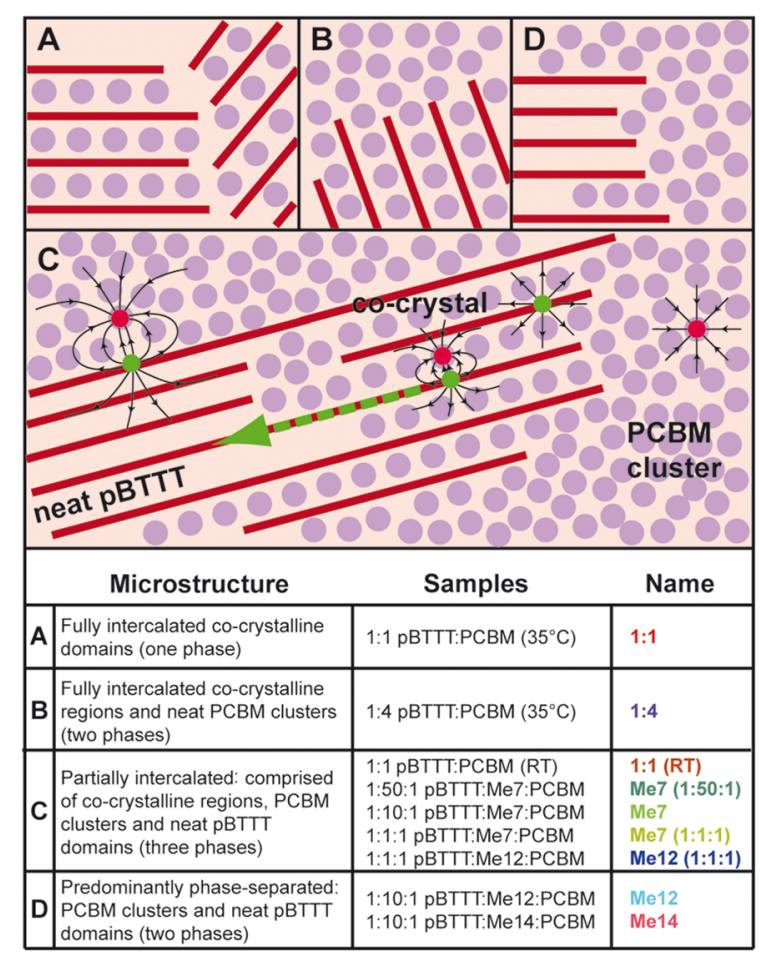

Figure 2. Schematic representation of the four investigated microstructures for pBTTT:PCBM, as described in the lower panel table. The different processing conditions yielding each microstructure are also summarized, together with the abbreviated sample names used in the main text (color coded according to the absorption spectra in Figure 1). Note that the molecules and domain sizes are not drawn to scale in the schematic representation. Please refer to Figures S2 and S3 in the Supporting Information for additional structural data and to ref 24 for details on the domain sizes and distribution.

Both the PCBM and the polymer signatures are present in the absorption spectrum of this blend (Figure 1B). While the fullerene displays a similar signature as in the neat system, the absorption of the polymer shows a pronounced structure due to a vibronic progression and is more red-shifted (maximum at $558 \mathrm{~nm}$ ) compared to the band in neat pBTTT. This spectral difference has been previously reported, ${ }^{20}$ and was attributed to significant changes in the crystal structure between neat and intercalated pBTTT (including changes in backbone conformation, chain stacking, and thus electronic coupling). ${ }^{49}$ Theoretical work for other conjugated polymers confirms the important implications of intra- and intermolecular coupling on the extent of vibronic contribution to the absorption spectrum. ${ }^{50}$ For the 1:4 pBTTT:PCBM blend (by weight), there is excess PCBM present, which forms relatively pure clusters around the intermixed regions, yielding a two-phase scenario (Figure 2B). ${ }^{20,22,48}$ As a consequence, the PCBM 
absorption is strongly enhanced, while the structured shape of the pBTTT band confirms that the polymer is still predominantly present in the co-crystalline domains (Figure 1B).

Processing the 1:1 blend at room temperature (RT) changes the structural picture compared to the same system cast at 35 ${ }^{\circ} \mathrm{C}$. The polymer exhibits in such films a broad absorption band around $540 \mathrm{~nm}$ and a shoulder at $605 \mathrm{~nm}$, indicating incomplete fullerene intercalation (coexistence of neat pBTTT and co-crystal regions, Figure 1B). A more reliable strategy to control the PCBM intercalation is to use asymmetrical processing additives, which direct the supramolecular assembly of the two components in the blend. ${ }^{24}$ The structure of heptanoic acid methyl ester (Me 7) and the absorption spectra of the 1:1 pBTTT:PCBM blend (by weight) processed with 1,10 , and $50 \mathrm{~mol}$ equiv of Me 7 per pBTTT monomer unit are depicted in Figure 1C. For the three samples, the superposed absorption of the broad and structured pBTTT features shows that the PCBM starts to phase separate from the polymer (partial fullerene intercalation). The relative weight of the broad $540 \mathrm{~nm}$ signature versus the $605 \mathrm{~nm}$ shoulder suggests that the phenomenon is slightly more pronounced for the lowest Me 7 concentration. The picture of partial intercalation is in agreement with our previous study, where a variety of tools (grazing-angle incidence wide-angle Xray scattering, scanning transmission X-ray microscopy, optical microscopy, resonant soft X-ray scattering) confirmed the three-phase microstructure in the 1:10:1 pBTTT:Me7:PCBM system. ${ }^{24}$ The concomitant presence of a significant fraction of co-crystal phase with some (relatively) neat PBTTT and PCBM regions is schematically shown in Figure 2C.

Our previous structural investigation also revealed that using additives with longer alkyl chains, such as dodecanoic acid methyl ester (Me 12, Figure 1D) or tetradecanoic acid methyl ester (Me 14) leads to further expulsion of PCBM from the cocrystal regions compared to using Me 7. A predominantly twophase microstructure comprised of relatively phase-pure polymer and fullerene domains is obtained (Figure 2D). ${ }^{24}$ The absorption spectra of the 1:1 pBTTT:PCBM blends processed with $\mathrm{Me} 12$ and $\mathrm{Me} 14$ show a pronounced structureless signature in the $540 \mathrm{~nm}$ region and only a weak shoulder at $605 \mathrm{~nm}$, confirming the predominant presence of neat polymer domains (Figure 1D). Moreover, there is a surprising decrease of the PCBM band below $400 \mathrm{~nm}$ for the samples containing $10 \mathrm{~mol}$ equiv of Me 12 or Me 14, although the amount of fullerene has in principle not been reduced.

To verify the PCBM concentration in those blends, we redissolved the Me 12 (1:10:1) sample in chlorobenzene, and indeed recovered the fullerene absorption to the same level as in redissolved 1:1 and Me 7 blends (Figure S4, Supporting Information). We also excluded film inhomogeneity as the origin of the observed effect, by moving the sample and using a large beam. Neither did we notice an enhancement of the relative polymer absorption, which might have explained the reduced PCBM peak in the normalized spectra. A similar reduction of PCBM absorption has been noted for annealed blends of poly(3-hexylthiophene) (P3HT) with PCBM. ${ }^{51}$ The effect is possibly related to enhanced long-range ordering in the neat PCBM regions of the Me 12 and Me 14 samples (as opposed to short-range aggregation obtained with $\mathrm{Me} 7)^{52}$ The presence of large PCBM-rich domains in the Me 12 and Me 14 samples is supported by structural data (Figures S2 and S3, Supporting Information, and ref 24). When less Me 12 was used (1:1:1 blend in Figure 1D), the relative amplitude of the PCBM signature remained high, suggesting that the phenomenon causing the reduced amplitude (presumably long-range PCBM ordering) is limited, as in the case of the blends processed with Me 7 .

2.2. Excited-State Dynamics of Neat pBTTT Films. The femtosecond TA spectra of a neat pBTTT film excited at 540 $\mathrm{nm}\left(<8 \mu \mathrm{J} / \mathrm{cm}^{2}\right)$ are shown in Figure 3A. The broad negative
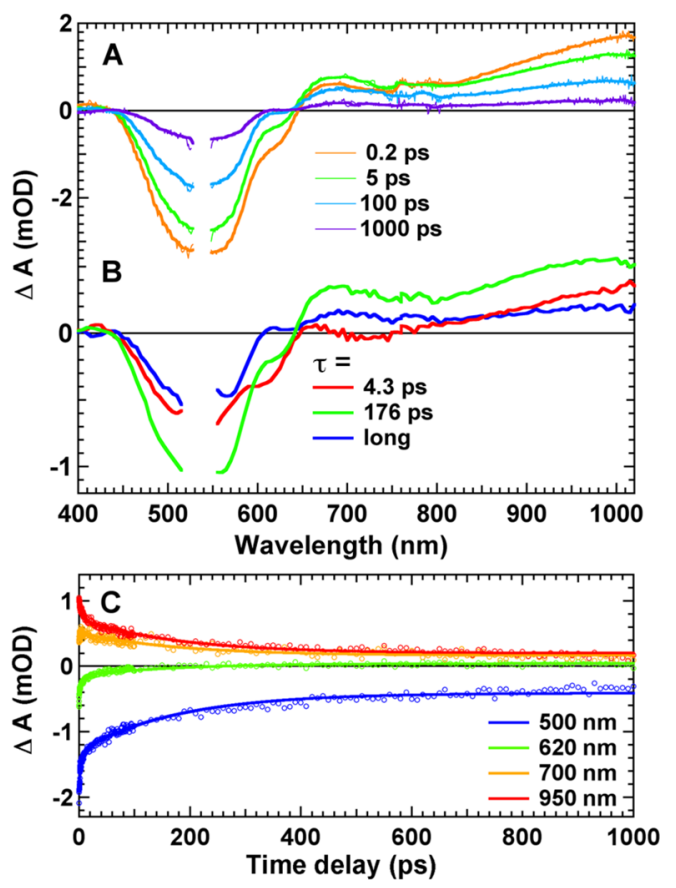

Figure 3. TA data for neat pBTTT films. (A) TA spectra recorded at selected time delays following $540 \mathrm{~nm}$ excitation. Thicker solid lines are smoothed and overlaid to the raw experimental data. (B) Amplitude spectra associated with the time constants (shown in the legend) obtained by multiexponential global analysis of the data. The "long" time constant is a plateau showing no decay within the measured time window. (C) TA dynamics at selected probe wavelengths. The solid lines represent the best multiexponential fit obtained by global analysis.

band around $530 \mathrm{~nm}$ is assigned to ground state bleaching (GSB) of the polymer. The singlet excited state (exciton) absorption (ESA) has a positive signature above $640 \mathrm{~nm}$, characteristically peaking toward $1000 \mathrm{~nm}$. Finally, the negative shoulder around $630 \mathrm{~nm}$ and the indent around $770 \mathrm{~nm}$ are due to stimulated emission (SE), which resembles the steadystate fluorescence spectrum. ${ }^{24}$ Inhomogeneity in the exciton population of conjugated polymers is typically caused by disorder, breaking the conjugation into chromophoric segments of different lengths and leading to multiphasic excited state behavior. ${ }^{53}$ It is therefore not surprising that the decay of the TA features for neat pBTTT films is biphasic, with a fast component and a slower component in the TA dynamics, shown for selected wavelengths in Figure 3C. They were analyzed globally with time constants of 4.3 and 176 ps, yielding respectively the red and green amplitude spectra in Figure 3B (see the Supporting Information for details on the fitting procedure). The complex excited state behavior is confirmed by the strongly multiphasic fluorescence dynamics, measured by femtosecond up-conversion spectroscopy upon $500 \mathrm{~nm}$ excitation (Figure 4A). Time constants of $0.4,4.9,62$, 
and 600 ps were necessary to globally reproduce the dynamics at emission wavelengths between 700 and $800 \mathrm{~nm}$.

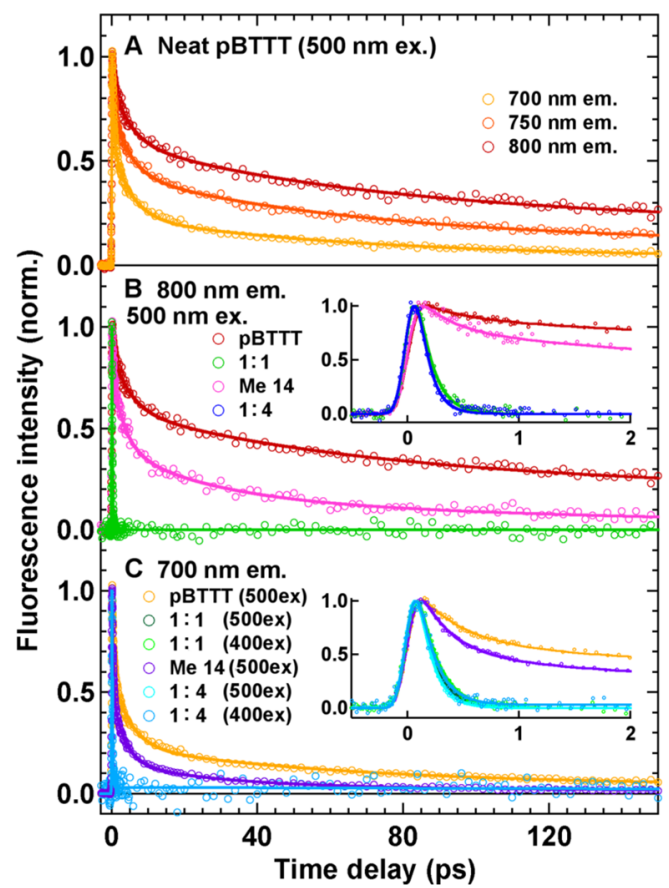

Figure 4. Femtosecond fluorescence dynamics obtained by the upconversion technique. (A) Neat pBTTT film at various emission wavelengths following excitation at $500 \mathrm{~nm}$. (B) Emission at $800 \mathrm{~nm}$ for neat and blend films (1:1 and 1:4, processed at $35^{\circ} \mathrm{C}$ ), following excitation at $500 \mathrm{~nm}$. (C) Emission at $700 \mathrm{~nm}$ for the neat and blend films, following excitation at 400 or $500 \mathrm{~nm}$. Solid lines are the best multiexponential fit from global analysis.

It can be seen from the data displayed in Figure 4A that the amplitude of the fast decay components is enhanced at shorter emission wavelengths, indicating faster decay on the blue side of the fluorescence spectrum. In general, this is typical for any kind of relaxation leading to a dynamic red-shift of the emission. ${ }^{54-56}$ In agreement, the shape of the 4.3 ps amplitude spectrum from the TA data shows decay of a blue-shifted SE signature. This kind of early relaxation in conjugated polymers is usually caused by excitation energy transfer to lower-energy chromophores, and possibly by geometrical changes in the polymer backbone. ${ }^{56-59}$ There is, however, pronounced ground-state recovery for pBTTT on the 4.3 ps time scale (see GSB contribution to the amplitude spectrum). This suggests that the high-energy polymer segments do not only transfer their excitation energy (which should maintain the GSB), but that they are also lost, at least partially, to the ground state on the very fast time scale. On the other hand, the $176 \mathrm{ps}$ component of the TA data corresponds essentially to the lifetime of relaxed low-energy pBTTT excitons with more redshifted SE (Figure 3B). At long time delays, a plateau (offset) is observed in the TA dynamics (the corresponding amplitude spectrum is denoted as "long"). It is due to long-lived TA features with negligible SE contribution and flat absorption above $600 \mathrm{~nm}$ (mainly charges and/or the triplet state)..$^{0,61}$

2.3. Charge Generation in pBTTT:PCBM Blends. 2.3.1. Fully Intercalated Samples. We discuss here the $1: 1$ and 1:4 pBTTT:PCBM blends processed at $35{ }^{\circ} \mathrm{C}$ (simply referred to as 1:1 and 1:4 samples in the following), which both have essentially complete fullerene intercalation between the polymer chains, with additional PCBM clusters for the 1:4 sample (as shown schematically in Figure 2A,B). The TA spectra in panels $\mathrm{A}$ and $\mathrm{C}$ of Figure 5 were obtained with
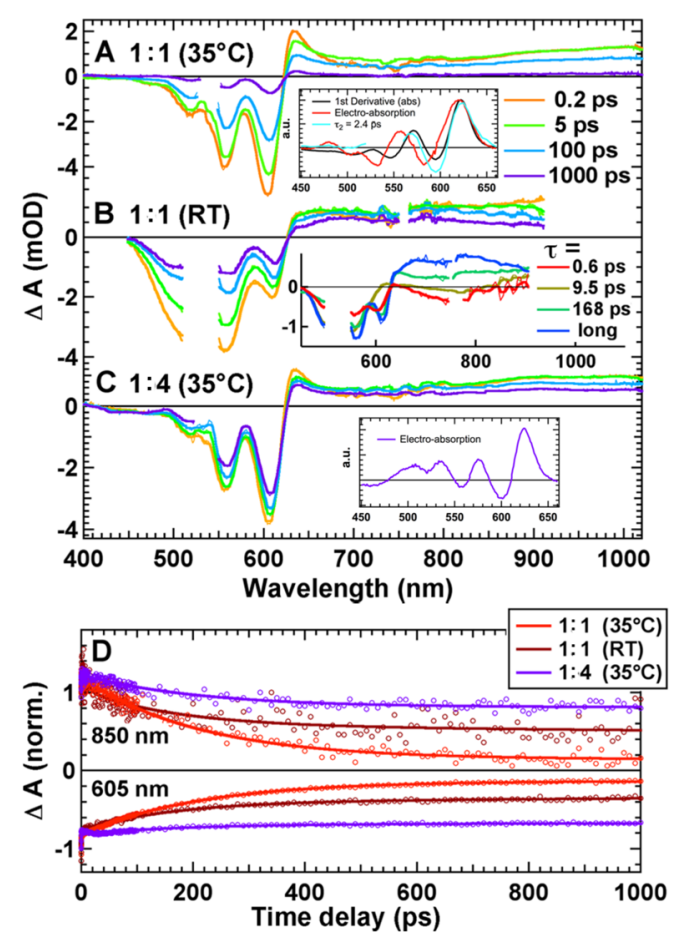

Figure 5. TA data for 1:1 and 1:4 pBTTT:PCBM films (by weight) following excitation at $540 \mathrm{~nm}$. (A) TA spectra at selected time delays for the fully intercalated $1: 1$ blend processed at $35^{\circ} \mathrm{C}$. The inset shows the first derivative of the steady-state absorption spectrum (black), the electro-absorption spectrum measured on a solar cell with an applied bias of $6 \mathrm{~V}$ (red), and the amplitude spectrum associated with the 2.4 ps time constant obtained by global analysis of the TA data (cyan); see Figure S7 in the Supporting Information for an enlarged version. (B) TA spectra of the partially intercalated 1:1 blend processed at room temperature (RT). The inset shows the amplitude spectra associated with the time constants obtained by multiexponential global analysis of the data. (C) TA spectra of the fully intercalated 1:4 blend processed at $35{ }^{\circ} \mathrm{C}$. The inset shows the electro-absorption spectrum measured on a solar cell with an applied bias of $6 \mathrm{~V}$ (violet); see Figure S7 in the Supporting Information for an enlarged version. For panels A-C, thicker solid lines are smoothed and overlaid to the raw experimental data. (D) TA dynamics probed at 605 and $850 \mathrm{~nm}$ following excitation at $540 \mathrm{~nm}$, for the two 1:1 blends and the 1:4 blend. Solid lines represent the best multiexponential fit.

excitation of mainly the polymer at $540 \mathrm{~nm}$. Corresponding spectra with $390 \mathrm{~nm}$ excitation of mainly PCBM are depicted in Figure S5 (Supporting Information), and all the amplitude spectra are recapitulated in Figure S6 (Supporting Information). Low fluence $\left(<10 \mu \mathrm{J} / \mathrm{cm}^{2}\right)$ was generally used and the absence of any annihilation effects or degradation was verified (Figure S1, Supporting Information). We have discussed the detailed TA analysis for the two samples elsewhere. ${ }^{48}$ In brief, all pBTTT excitons in the 1:1 and 1:4 blends dissociate into charges on the ultrafast $\sim 100$ fs time scale, given the proximity of the polymer and fullerene in the co-crystal phase (no exciton diffusion is necessary).

The time-resolved emission dynamics with $500 \mathrm{~nm}$ excitation (Figure 4B) confirm this prompt quenching of the $800 \mathrm{~nm}$ polymer fluorescence within 130 and 110 fs for the $1: 1$ and $1: 4$ 

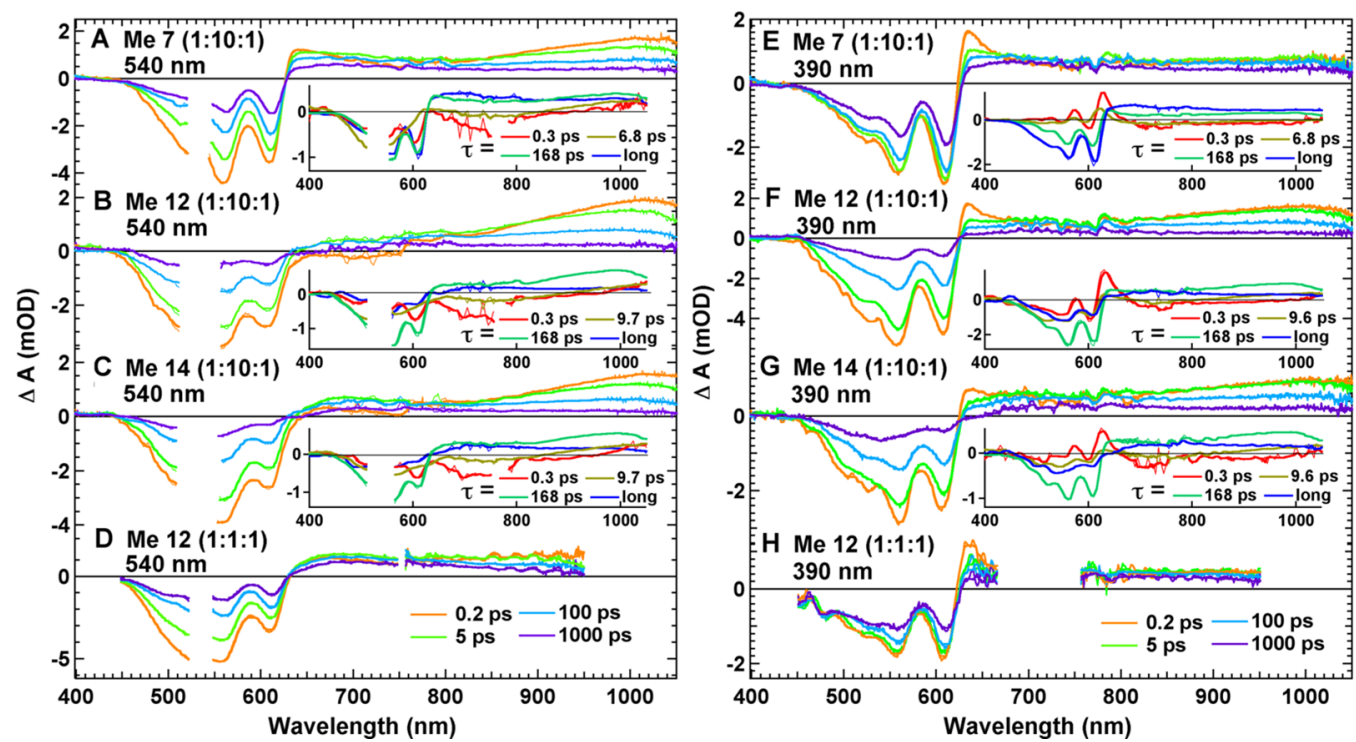

Figure 6. TA spectra at selected time delays for 1:1 pBTTT:PCBM films processed with Me 7, Me 12, and Me 14 additives, following excitation at $540 \mathrm{~nm}(\mathrm{~A}-\mathrm{D})$ or at $390 \mathrm{~nm}(\mathrm{E}-\mathrm{H})$. The insets represent the amplitude spectra associated with the time constants obtained by multiexponential global analysis of the data. Thicker solid lines are smoothed and overlaid to the raw experimental and amplitude spectra.

blend, respectively (a convolution with the $100 \mathrm{fs}$ instrument response function was used and it is likely that even faster quenching components are missed). The higher-energy emission at $700 \mathrm{~nm}$ (Figure 4C) also decays with $150 / 180 \mathrm{fs}$ (1:1 blend) and 130/150 fs ( $1: 4$ blend), following excitation at $500 / 400 \mathrm{~nm}$. With $400 \mathrm{~nm}$ excitation, there is a weak contribution of PCBM emission, ${ }^{52}$ which is quenched by hole transfer (HT) to pBTTT. Our previous TA study has revealed this process to be prompt in the 1:1 blend ( $\sim 100 \mathrm{fs})$, but to have a distribution of ultrafast to slow components (ranging from sub-ps to $\sim 500 \mathrm{ps}$ ) in the 1:4 blend due to diffusion of PCBM excitons to a quenching site through the fullerene clusters (see amplitude spectra in Figure S6, Supporting Information). ${ }^{48}$ This causes the weak offset at long time delays in the emission dynamics of the 1:4 blend excited at $400 \mathrm{~nm}$ (Figure 4C).

For the fully intercalated 1:1 blend, it is known that most electron-hole pairs generated by CS recombine geminately to the ground state, most likely because they cannot overcome their mutual Coulomb attraction. ${ }^{20,22,24,28,62}$ This is consistent with the low solar cell efficiency of this system. Here, we directly observe the geminate charge recombination ( $\mathrm{gCR}$ ) as a significant decay of all TA features with a relatively short $211 \mathrm{ps}$ time constant (Figure 5A, dynamics of the $605 \mathrm{~nm} \mathrm{GSB}$ and $850 \mathrm{~nm}$ charge absorption in Figure 5D, amplitude spectra in Figure S6, Supporting Information). ${ }^{48}$ Only a small fraction of free charges is formed, leading to a weakly pronounced longlived TA spectrum at our longest time delay of $1 \mathrm{~ns}$. In the 1:4 blend, the presence of PCBM clusters largely suppresses $\mathrm{gCR}^{20,22,28,62}$ so that the decay of the TA features on the $1 \mathrm{~ns}$ time scale is notably reduced (Figure 5C,D). This can be explained by favored spatial separation of charge pairs formed in the co-crystalline regions, driven by an energy cascade to neat fullerene domains with higher electron affinity. ${ }^{22,37}$ The charge dissociation efficiently competes with the 211 ps recombination process, so that we infer that the electron migration to the PCBM clusters is faster than the $\mathrm{gCR}(<211$ ps). Electrons are thus largely removed from the co-crystal phase within the time window of the experiment.
2.3.2. Partially Intercalated Samples. This section concerns samples having a three-phase microstructure (partial fullerene intercalation), comprised of co-crystalline regions, PCBM clusters, and neat pBTTT domains, as schematically depicted in Figure 2C (1:1 pBTTT:PCBM blends processed at RT, with different amounts of Me 7, or with 1 mol equiv of Me 12). For the $1: 1$ blend processed at RT, which we will refer to as $1: 1$ (RT), the TA spectra with $540 \mathrm{~nm}$ excitation (Figure 5B) are clearly different from the fully intercalated 1:1 sample. In particular, the amplitude of the long-lived TA features is significantly enhanced, pointing to a microstructure that favors generation of free charges. The dynamics in Figure 5D confirm the reduction of gCR (although still more charges recombine than in the 1:4 blend). In agreement with the similarity of the steady-state absorption, the TA behavior of the 1:1 (RT) sample (Figure 5B) is comparable to the one of the 1:1 blend processed with Me 7, which also results in partial fullerene intercalation (shown for $10 \mathrm{~mol}$ equiv and $540 \mathrm{~nm}$ excitation in Figure 6A). For convenience, we will simply call the latter the Me 7 sample. As with the 1:1 (RT) sample, processing of the 1:1 blend with Me 7 reduces the extent of gCR (see charge dynamics at $850 \mathrm{~nm}$ in Figure 7C). The partial phase separation of the polymer and fullerene due to the addition of Me 7 has also previously been correlated to enhanced free charge yield (by nanosecond TA spectroscopy and time-resolved microwave conductivity) and to improved solar cell efficiency compared to fully intercalated systems. ${ }^{24}$

For both partially intercalated samples (RT and Me 7), the GSB contains an unstructured contribution in the $530 \mathrm{~nm}$ region, which we attribute to the presence of neat $\mathrm{pBTTT}$ domains (Figures 5B and 6A). Since neat pBTTT domains and co-crystalline regions are excited at $540 \mathrm{~nm}$, we expect prompt CS in the highly intermixed regions as well as multiphasic delayed CS for excitons diffusing (by various distances) through the neat regions toward PCBM. ${ }^{33-35}$ The characteristic ESA signature at $1000 \mathrm{~nm}$ in the early TA spectra (in addition to the flat absorption of promptly generated charges) confirms the delayed presence of pBTTT excitons in the neat domains. Their quenching leads to fast components in the TA dynamics, 

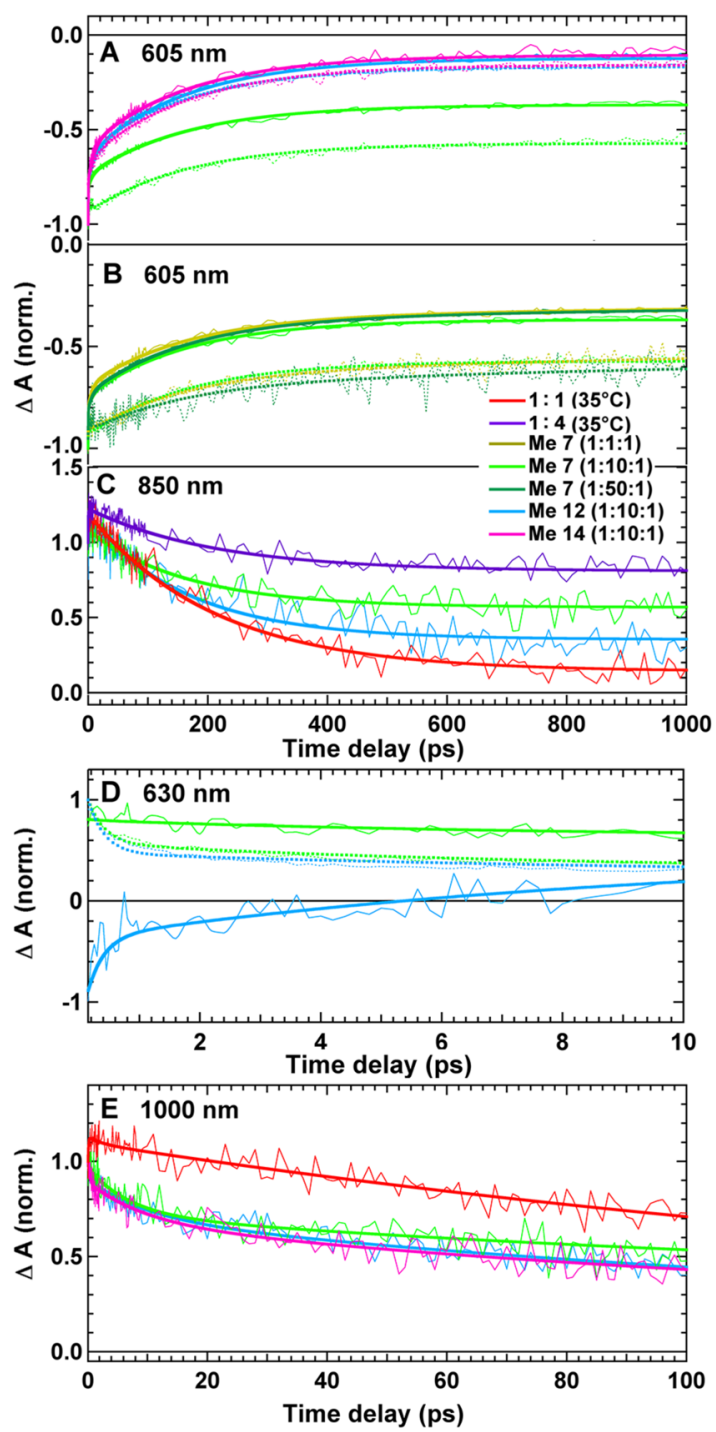

Figure 7. TA dynamics recorded for various blends shown in the legend at probe wavelengths of 605 (A, B), 850 (C), 630 (D), and $1000 \mathrm{~nm}(\mathrm{E})$. Solid lines represent $540 \mathrm{~nm}$ excitation, while dotted lines represent $390 \mathrm{~nm}$ excitation. The thicker lines are the best multiexponential fits from global analysis.

for example in the ESA at $1000 \mathrm{~nm}$ (Figure 7E, only slow decay of overlapping charge absorption is seen for the $1: 1$ blend). By global analysis, we find a subpicosecond time constant $(0.6 / 0.3$ ps for RT/Me 7) and a slower (9.5/6.8 ps) time constant for the delayed exciton quenching. This is assigned based on the decay of the negative SE around $730 \mathrm{~nm}$ and positive ESA above $870 \mathrm{~nm}$ in the corresponding amplitude spectra (insets of Figures 5B and 6A). We cannot exclude some slower exciton quenching masked by the evolution of the charge absorption. The amplitude spectra assigned to exciton quenching also indicate decrease of the GSB. Reduction of the GSB during CS has been reported in other polymer:fullerene blends. ${ }^{34,63}$ However, given the $\sim 4$ ps exciton decay in neat pBTTT films (Figure 3), it is possible that excitons in the neat regions are not only dissociated by delayed CS, but are also partly lost to the ground state. Finally, we observe a slower decrease of the TA features with a 168 ps time constant for the 1:1 (RT) and Me 7 samples, which we attribute to $\mathrm{gCR}$ in the co-crystalline regions, given the similarity of the amplitude spectrum with the one obtained for gCR in the 1:1 and 1:4 blends (Figure S6, Supporting Informatioin). ${ }^{48}$

Let us now turn to the TA data with $390 \mathrm{~nm}$ excitation, where both the polymer and the PCBM are excited. TA spectra for the partially intercalated 1:1 (RT) and Me 7 samples are shown in Figure S5 in the Supporting Information and Figure $6 \mathrm{E}$, respectively. In those blends, the PCBM is present both in the intercalated, co-crystalline phase, as well as in the relatively phase-pure fullerene clusters (as revealed by the presence of mobile electrons using time-resolved microwave conductivity). ${ }^{24} \mathrm{~A}$ complex overlap of prompt and delayed electron and hole transfer processes with recombination and spatial charge separation is therefore expected. The TA evolution is very similar for the 1:1 (RT) and Me 7 blends, but there are significant differences compared to the data obtained with 540 $\mathrm{nm}$ excitation. Most notably, there is an apparently reduced decay of the TA features for the $390 \mathrm{~nm}$ data (Figure S5, Supporting Information and Figure 6E). This is also obvious in the TA dynamics probed at $605 \mathrm{~nm}$ for the Me 7 sample (Figure 7A, green solid lines for $540 \mathrm{~nm}$ and dotted lines for $390 \mathrm{~nm}$ excitation). Since free charge yield in OPV blends is generally not increased with excess excitation energy, it is unlikely that gCR is reduced at $390 \mathrm{~nm} .{ }^{44}$ Indeed, the $168 \mathrm{ps}$ amplitude spectrum in the inset of Figure $6 \mathrm{E}$ shows that recombination is present with $390 \mathrm{~nm}$ excitation. A more likely interpretation is that delayed HT from PCBM clusters (as observed in the 1:4 blend, Figure S5 in the Supporting Information) leads to a rise of the polymer GSB and charge absorption, which partly counterbalances the decrease of the TA features due to recombination.

2.3.3. Predominantly Phase-Separated Samples. More phase-separated systems can be obtained when the 1:1 pBTTT:PCBM blends are processed with 10 mol equiv of Me 12 and Me 14, leading to relatively phase-pure domains of the polymer and fullerene (Figure 2D). ${ }^{24}$ The TA spectra of those samples (abbreviated as $\mathrm{Me} 12$ and $\mathrm{Me} 14$ in the following) are shown in Figure 6B,C for $540 \mathrm{~nm}$ excitation and Figure $6 \mathrm{~F}, \mathrm{G}$ for $390 \mathrm{~nm}$ excitation. The free charge yield and solar cell efficiency are less than for the three-phase microstructure in the Me 7 sample, but higher than in the fully intercalated 1:1 blend. ${ }^{24}$ This trend is clearly reflected by the TA dynamics probed at $850 \mathrm{~nm}$ (mainly charge absorption, Figure $7 \mathrm{C}$ ). The most long-lived signal is observed for the efficient 1:4 blend, followed by samples processed with Me 7, then Me 12, and finally the fully intercalated 1:1 blend. Thus, it is clear that recombination processes on the subnanosecond time scale determine the performance of $\mathrm{pBTTT}$ :PCBM devices.

There is no significant difference in the TA data between the blends processed with Me 12 and Me 14 (blue and pink curves in Figure 7A). With $540 \mathrm{~nm}$ excitation of pBTTT, the early 0.2 ps TA spectrum is dominated by signatures of pBTTT excitons (negative SE at $730 \mathrm{~nm}$ and positive ESA at $1000 \mathrm{~nm}$, Figure $6 \mathrm{~B}, \mathrm{C})$, without significant contribution of charge absorption. This strong reduction of prompt CS, as well as the more unstructured shape of the GSB, confirm the predominance of neat $\mathrm{pBTTT}$ domains rather than highly intermixed, cocrystalline regions. According to the fluorescence dynamics for 700 and $800 \mathrm{~nm}$ emission, the excitons in Me 14 blends are quenched more rapidly than in neat pBTTT films (Figure $4 \mathrm{~B}, \mathrm{C})$. Still, the quenching is quite slow and strongly multiphasic, with time constants of $290 \mathrm{fs}, 3.4 \mathrm{ps}, 25 \mathrm{ps}$, and $165 \mathrm{ps}$, confirming that exciton diffusion limits the CS rate. In 
the TA spectra, the exciton quenching contributes to the 0.3 and 9.7 ps amplitude spectra (insets of panels B and C in Figure 6; their shape is similar to the one obtained for the Me 7 sample). There is also a dominant contribution of exciton decay to the 168 ps component (see similarity with the 176 ps amplitude spectrum in neat pBTTT film, Figure 3B). For the fully intercalated (1:1 and $1: 4)$ and partially intercalated (1:1 (RT), Me 7) blends, the TA decay on this time scale is mainly caused by gCR of charge pairs in the co-crystal phase. For the more phase-separated Me 12 and Me 14 samples, the loss mechanism is different and results mainly from the decay of excitons in neat pBTTT domains to the ground state, which cannot reach a PCBM acceptor during their lifetime.

For the Me 12 and Me 14 samples, it is still predominantly the pBTTT which absorbs with excitation at $390 \mathrm{~nm}$, since there is very little absorption of PCBM in this region (fullerene light harvesting is lost due to long-range ordering of PCBM, Figure 1D). This explains the negligible difference in the dynamics with 540 and $390 \mathrm{~nm}$ excitation (solid and dotted lines in Figure 7A). The TA spectra with $390 \mathrm{~nm}$ excitation also exhibit a strong contribution of polymer excitons, undergoing slow multiphasic quenching and decay (Figure 6F,G). Our structural picture is further confirmed when using lower quantities of Me 12 during processing (1:1:1 blend), which allows us to restore a three-phase microstructure with significant PCBM absorption at $390 \mathrm{~nm}$. Exciton recombination losses are in this case reduced and the evolution of the TA spectra resembles the one of the 1:1 (RT) and Me 7 samples (Figure 6D,H).

2.4. Electro-absorption and Spatial Charge Separation. The photogenerated charges in polymer:fullerene blends create important electric fields in their vicinity, which can have strong effects on the optical transitions of neighboring chromophores. ${ }^{38}$ In general, an electric field $(E)$ perturbs the absorption spectrum of a molecule by shifting the energy levels of the ground and/or excited state (Stark effect), by breaking the symmetry allowing otherwise forbidden transitions, or by ionizing the excited state. ${ }^{64} \mathrm{We}$ focus here on the Stark effect, which occurs if there is a change of permanent dipole moment $(\Delta p)$ and/or of polarizability $(\Delta \alpha)$ between the ground and excited states, leading to an energy shift of the transition. The electro-absorption (EA) is defined as the difference of the absorption spectrum in the presence $\left(A^{\mathrm{E}}(\nu)\right)$ and absence $(A(\nu))$ of the electric field. It can be expressed as a series of first, second, and higher order derivatives of $A(\nu):^{64}$

$$
\begin{aligned}
\mathrm{EA}= & A^{E}(\nu)-A(\nu) \\
= & -\frac{\partial A(\nu)}{\partial \nu} \Delta p E-\frac{1}{2} \frac{\partial A(\nu)}{\partial \nu} \Delta \alpha E^{2}+\frac{1}{2} \frac{\partial^{2} A(\nu)}{\partial \nu^{2}}(\Delta p E)^{2} \\
& +\ldots
\end{aligned}
$$

The first term (linear in E) cancels in isotropic media, the second term (quadratic in $E$ ) shows that changes in polarizability are related to the first derivative of the absorption spectrum, and the third term associates changes in the permanent dipole moment to the second derivative of $A(\nu)$.

In TA spectroscopy, the absorption difference $(\Delta A)$ of the sample with and without excitation by the pump is measured, thus in the presence and absence of photogenerated charges in the case of the PBTTT:PCBM blends. If a Stark effect is caused by those charges, an EA contribution is seen in the TA data. In contrast to the case where a uniform electric field is externally applied across the bulk of the film using electrodes, ${ }^{43,65,66}$ the
Stark effect in the TA data depends on the local radial electric fields around free charges and the local electric dipoles around electron-hole pairs. It can be observed even if the overall macroscopic field cancels due to a random orientation of the electron-hole pairs in the $\mathrm{BHJ}$ (in particular for a quadratic dependence on the local field magnitude). ${ }^{38}$ The EA signature in the TA data can be exploited to determine in which phase of the blend the charges are generated and how they spatially separate. Key parameters determining the amplitude of the observed EA signal include the electron-hole distance, the orientation of the electric dipoles with respect to the crystal structure, and the nature of the chromophores found in the vicinity of the charges (neat or in the co-crystal phase). Some examples of electric field scenarios encountered in a threephase microstructure of pBTTT:PCBM are schematically illustrated in Figure 2C.

2.4.1. Fully Intercalated Samples. We have demonstrated that the electron transfer (ET) from photoexcited pBTTT to intercalated PCBM molecules is prompt for the 1:1 and 1:4 blends excited at $540 \mathrm{~nm}$. Therefore, only the signature of charges (not of excitons) is present in the TA spectra of Figure $5 A, C$. In addition to the expected GSB below $620 \mathrm{~nm}$ and the flat absorption of positively charged pBTTT above $620 \mathrm{~nm}$, an oscillatory EA signal resembling the first derivative of the absorption spectrum (inset of Figure 5A) superposes to the red side of the GSB and displays a characteristic positive peak around $630 \mathrm{~nm}$. We have confirmed this assignment by directly measuring the EA spectra of the 1:1 and 1:4 films sandwiched between electrodes in solar cell devices, with an externally applied uniform electric field of the order of $6 \times 10^{5} \mathrm{~V} / \mathrm{cm}$ (insets of panels $\mathrm{A}$ and $\mathrm{C}$ in Figure 5, experimental details in the Supporting Information). We note that the EA signature that is most visible in the TA spectra (600-650 nm region) results from transitions in pBTTT, while the weaker contribution of PCBM $(450-550 \mathrm{~nm})^{66}$ is masked by the GSB of the polymer. We will therefore limit the discussion to the EA signal induced by photogenerated charges in the polymer chains. The EA amplitude measured in the device at $620 \mathrm{~nm}$ varies quadratically with the externally applied bias (Figure S8B, Supporting Information). We are thus observing a quadratic Stark effect (the transition shifts with $E^{2}$ ). Since the EA spectrum of the 1:1 blend shows similarity to the first derivative of the absorption (Figure 5A and Figure S7 in the Supporting Information), the main origin of the Stark effect is a change in polarizability between the ground and excited state of pBTTT.

It is noteworthy that the EA signature observed in the TA data of the 1:1 blend (upon $390 \mathrm{~nm}$ or $540 \mathrm{~nm}$ excitation) is instantaneously present at the shortest time delays (Figure 5A and Figure S5A in the Supporting Information), although the charges generated by prompt CS in this highly intermixed, fully intercalated system must be in close proximity and are presumably significantly bound. Different behavior has been reported for other systems, where the EA signal was only associated with long-range $(4-5 \mathrm{~nm})$ separated charges, but absent in samples with low fullerene loading and strong electron-hole binding. ${ }^{38}$ We relate this to the significantly different microstructure of the pBTTT:PCBM blend, which forms a well-defined co-crystal phase, compared to the molecularly less ordered polymer and small molecule systems discussed in ref 38.

Indeed, we can explain our observations in the 1:1 blend using simple electrostatic considerations applied to the 
particular geometry of the pBTTT:PCBM co-crystal phase (see the Supporting Information for details and Figure S9 for the published crystal structure $\left.{ }^{49}\right)$. Since the local field generated by a charge decays with distance according to $1 / a^{2}$ (and $E^{2}$ decays even faster with $\left.1 / a^{4}\right)$, only the pBTTT chromophores located in closest proximity to a charge will experience a significant Stark effect. In the co-crystal phase, the initially generated dipole related to the electron-hole pair is perpendicular to the pBTTT backbones (Figure 2C). By inspection of the crystal structure shown in Figure S9, Supporting Information, the polymer segments closest to a charge (which will dominate the EA response) are the ones located on both sides of the hole on the same chain, and the ones on the chains $\pi$-stacked above and below the hole. The field generated by the hole across the lamella spacing of $\sim 3 \mathrm{~nm}$ is much weaker. ${ }^{49}$ Similarly, the effect of the field around the electron is small on the pBTTT chromophores, as it will mainly affect the close-by fullerene molecules. Electrostatic simulations show that the field felt by the relevant polymer segments next to the hole vanishes if the electron-hole distance approaches zero (see the Supporting Information). However, if we assume an initial charge separation distance of $1 \mathrm{~nm}$ (which is reasonable based on the size and arrangement of the pBTTT and PCBM molecules), ${ }^{38}$ the field at the relevant positions is already significant and explains the presence of the EA signature in the TA spectra of the 1:1 blend at early time delays. In fact, the polymer segments located at a distance of about $0.2-0.5 \mathrm{~nm}$ away from the hole in the co-crystal geometry experience an electric field very close to the one that would be created around a free hole.

Our previous analysis of the TA data for the intercalated 1:1 blend has revealed that the only evolution of the EA signature is a weak decrease with a 2.4 ps time constant. ${ }^{48}$ The corresponding amplitude spectrum indeed strongly resembles the EA signature (inset of Figure 5A and Figure S6, Supporting Information). In spite of this weak decay, the $630 \mathrm{~nm}$ EA peak is still clearly visible at the longest ( $1 \mathrm{~ns}$ ) time delay. We tentatively ascribe the EA decay to some relaxation of the charges involving change in their delocalization, or to their migration within the co-crystal phase (the electrostatic simulations in the Supporting Information confirm that the associated electron-hole separation and rotation of the dipole with respect to the crystal frame could lead to a decrease of the EA). In the 1:1 blend, migration away from their initial generation site concerns only a small fraction of escaped charges, since most of them undergo gCR on the 200 ps time scale. In contrast, the presence of neat PCBM clusters in the 1:4 blend provides a driving force for spatial charge separation and significantly prevents recombination within the time window of our experiment. ${ }^{22}$ Thereby, the electron transport within the co-crystalline regions occurs in the direction parallel to the polymer $\pi$-stacks, because it is hindered in the other two directions by the polymer backbones and pBTTT side chains (Figure S6, Supporting Information). ${ }^{49}$

One would expect a different evolution of the EA signature in the $1: 4$ sample, but it is surprisingly similar to the one of the $1: 1$ blend without neat fullerene regions. The EA appears in both cases instantaneously upon prompt CS in the co-crystal phase, then slightly decays within $2.4 \mathrm{ps}$, and remains significantly present for free charges at long time delays (Figure 5, and in the Supporting Information Figures S5 and S6). We can again explain this observation by considering the molecular geometry around the photogenerated charges (Figure 2C). For the initial electron-hole pair in the co-crystal phase (separated by $1 \mathrm{~nm}$ ), the pBTTT segments most affected by the charges are close to the hole $(<0.5 \mathrm{~nm})$ and in the plane perpendicular to the dipole (similar as discussed above for the 1:1 blend). According to electrostatic considerations (see the Supporting Information), they experience an electric field comparable to the one around a free hole (i.e., the influence of the electron is negligible at this position). There is therefore not a large difference in the EA amplitude when the charges effectively separate to a distance of $5 \mathrm{~nm}$ thanks to the PCBM clusters (Figure 2C). A significantly stronger dependence of the EA on the electron-hole separation would occur if the closest neighbors to the hole in the co-crystal phase were along the axis of the initial charge dipole, but this is not the case for the co-crystal geometry. The Stark effect observed for intercalated pBTTT:PCBM samples is thus quite independent of whether the electrons stay in the cocrystalline regions or not, but it is dominated by the presence of (bound or free) holes on polymer chains within the co-crystal regions.

2.4.2. Partially Intercalated and Predominantly PhaseSeparated Samples. For the partially intercalated 1:1 (RT) and Me 7 blends excited at $540 \mathrm{~nm}, \mathrm{gCR}$ is reduced compared to the fully intercalated 1:1 blend, which we attribute to the presence of phase-pure domains in the former blends, similar to the 1:4 system. However, neat $\mathrm{pBTTT}$ regions are now present in addition to the PCBM clusters. ${ }^{24}$ The TA spectra recorded at long time delays ( $1 \mathrm{~ns})$ predominately represent the signature of long-lived free charges (Figures 5B and 6A). The GSB and flat absorption of positively charged $\mathrm{pBTTT}$ are obvious, but there is a striking absence of the EA signal peaking at $630 \mathrm{~nm}$. The EA signal is weakly present in the 0.2 ps spectrum, but almost disappears within a few picoseconds. Since we have seen for the 1:4 blend that the EA amplitude is quite insensitive to the migration of electrons to PCBM clusters, we deduce that the fast decay of the EA in the partially intercalated samples is related to the hole migration from the co-crystal phase to the neat pBTTT domains. Following a similar reasoning based on electrostatic simulations as for the 1:4 blend, we show in the Supporting Information that increasing the electron-hole separation by itself should not have a strong effect on the field experienced by the close neighbors of the hole. The strong reduction of the EA in the partially intercalated samples is therefore rather related to the nature of the polymer segments surrounding the hole. Indeed, the electronic properties of pBTTT are very different when it is in the neat domains or cocrystal phase, as testified by the change in the absorption spectrum (Figure 1A,B). The less steep absorption edge in the broad spectrum of neat $\mathrm{PBTTT}$ leads to a smaller value of the first derivative and thus to weaker EA. Experimentally, the EA spectrum recorded for a device containing neat pBTTT (with externally applied field) confirms that it is less sharp and reduced compared to the intercalated 1:1 blend (Figure S8A, Supporting Information). Thus, the electric field generated by a hole in neat pBTTT domains will induce a weak EA in neighboring polymer segments, not visible in the TA spectra.

Further evidence that the prominent EA signature is specifically related to holes in co-crystalline regions is shown in Figure $8 \mathrm{~A}-\mathrm{C}$ (540 $\mathrm{nm}$ excitation). Here, the concentration of Me 7 was varied during processing of the 1:1 blend, allowing slight tuning of the extent of PCBM intercalation. The early oscillatory EA signature due to prompt CS is more pronounced in the 1:50:1 blend containing more co-crystal fraction, than in the less intercalated 1:1:1 blend (in addition to weak changes in 


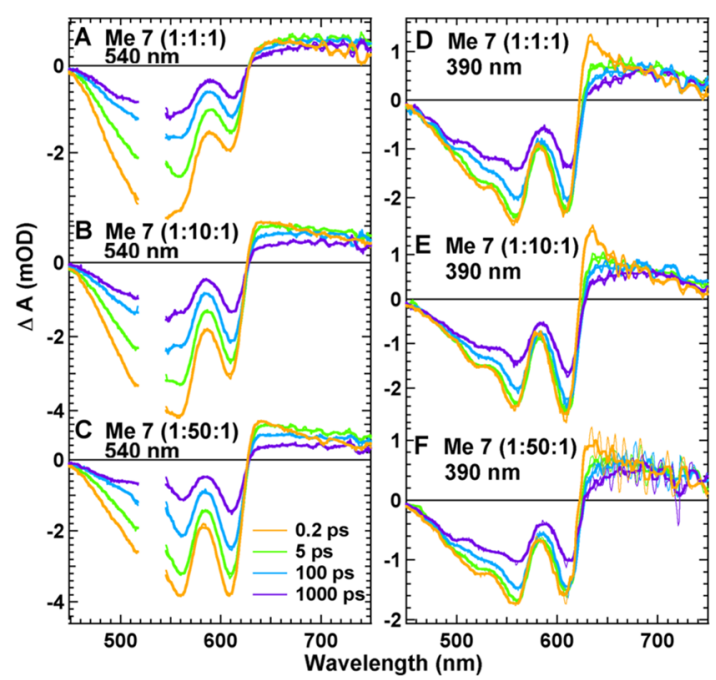

Figure 8. TA spectra at selected time delays for 1:1 pBTTT:PCBM films processed with different amounts of Me 7 additive, following excitation at $540 \mathrm{~nm}(\mathrm{~A}-\mathrm{C})$ or at $390 \mathrm{~nm}(\mathrm{D}-\mathrm{F})$. Thicker solid lines are smoothed and overlaid to the raw experimental data and raw amplitude spectra.

the GSB shape according to the steady-state absorption). Apart from this initial difference, the TA dynamics and overall evolution of the TA spectra is similar for the three samples (Figure 7B).

Although it has been previously established that neat domains in the $\mathrm{BHJ}$ favor the spatial separation of charge pairs generated in intermixed regions, ${ }^{20-22,48}$ a direct visualization of charge migration between different phases has to our knowledge not been reported. With the phase-dependent EA signature in pBTTT, we have a unique tool in hand to directly monitor the generation site of charges and the migration of holes from co-crystalline to neat polymer regions. Important conclusions can be drawn for the partially intercalated and more phase-separate pBTTT:PCBM blends:

(1) The presence of the $630 \mathrm{~nm}$ EA peak at ultrafast times designates co-crystal regions as the prompt charge generation site in the blends containing a significant intermixed fraction (1:1 blends processed at RT and with 10 or $50 \mathrm{~mol}$ equiv of $\mathrm{Me}$ 7 , excited at $540 \mathrm{~nm}$ ). Prompt CS can also occur for excitons formed at the domain boundaries of neat phases (no diffusion is necessary and the hole is immediately present in the neat domain, Figure 2C), leading to an initial charge signature without a visible EA signal (less intercalated Me 7 (1:1:1) and Me $12(1: 1: 1)$ samples, excited at $540 \mathrm{~nm})$. Since there is in general no obvious rise of the EA during delayed CS in the partially intercalated samples as well as in the more phaseseparated Me 12 and Me 14 samples (Figure 6B,C), this process also seems to occur at the interfaces between phasepure domains.

(2) Upon $390 \mathrm{~nm}$ excitation of all the partially intercalated samples (1:1 RT, Me 7) as well as the more phase separated Me 12 and Me 14 systems, there is a strong enhancement of the oscillatory EA signature in the early 0.2 ps spectra (Figure $6 \mathrm{E}-\mathrm{H}$ and Figure S5B, Supporting Information). This suggests a selective excitation of PCBM and possibly pBTTT in the cocrystal phase at $390 \mathrm{~nm}$. The effect is independent of the Me 7 concentration used during processing, confirming that promptly generated holes (with $390 \mathrm{~nm}$ excitation) are in all cases predominantly found in the highly intermixed, interca- lated regions (Figure 8D-F). The signature of free charges at long time delays is the same for both excitation wavelengths (without EA signal).

(3) Whenever charges are promptly generated in the cocrystal phase for the partially intercalated systems (with 540 and $390 \mathrm{~nm}$ excitation) and for the predominantly phaseseparated blends (with $390 \mathrm{~nm}$ excitation), the holes migrate to neat $\mathrm{pBTTT}$ regions on the $\sim 1$ ps time scale, as evidenced by the fast decay of the EA signature. For $390 \mathrm{~nm}$ excitation, this is clearly seen in the dynamics of Figure 7D. The EA decay is also reproduced by the oscillatory shape of the 0.3 ps amplitude spectra (insets of Figure $6 \mathrm{E}-\mathrm{G}$ ), and might contribute to the slower 6.8 or 9.6 ps components, together with a rise of the GSB due to delayed HT. The EA decay is less obvious in the amplitude spectra with $540 \mathrm{~nm}$ excitation, because of less selective excitation of the co-crystal phase and mixing with the ET dynamics.

(4) The results confirm that neat polymer domains in partially intercalated samples favor spatial separation of charge pairs generated in the intermixed co-crystal phase, contributing to reduced gCR, in the same way as PCBM clusters in the 1:4 blend. They can, however, lead to loss of short-lived pBTTT excitons. From the $\sim 1$ ps decay of the EA, we conclude that the holes can move immediately after their generation and do so very quickly. In agreement with recent Monte Carlo simulations, ${ }^{37}$ we suggest that this fast transport is sustained by high local mobility and by an energy cascade driving the holes to the neat regions. An energy cascade of $100-200 \mathrm{meV}$ has been reported for the electron transport to the fullerene clusters, ${ }^{22}$ and can also be expected between the intermixed and neat $\mathrm{pBTTT}$ regions, given the different electronic properties of the polymer in the two phases (see change in the absorption spectrum). Within the co-crystal phase, a hole can in principle migrate along the polymer backbones or along the $\pi$-stacking direction, but the former seems to be favored. ${ }^{67}$ We therefore hypothesize that the ultrafast hole migration occurs predominantly along the same polymer chains, which extend from the co-crystalline to neat pBTTT domains (so-called "tie"molecules, Figure 2C). ${ }^{68}$ By probing the environment of the charge, the Stark effect is therefore more useful to determine the location of the hole than the GSB, which probes the charged polymer chain itself.

(5) For the Me 7 and 1:1 (RT) samples (three phase microstructure), the presence of the $630 \mathrm{~nm}$ EA signature in the 168 ps amplitude spectrum assigned to gCR suggests that this recombination is dominant for holes remaining in the intermixed co-crystal regions (insets of Figures 5B and 6A,E). The charges are likely to be trapped in the intermixed phase due to poor (through-chain) connectivity to neat pBTTT regions and missing percolated paths to PCBM clusters. ${ }^{21,68}$

\section{CONCLUSION}

We have demonstrated a precise correlation between the early charge generation mechanism and the phase morphology of polymer:fullerene bulk heterojunction blends. Control of the charge separation was achieved by targeting well-defined microstructures through the use of fatty acid methyl esters processing additives. The rate of electron and hole transfer was determined by femtosecond absorption and emission spectroscopy, while an electro-absorption (Stark effect) signature allowed us to directly visualize ultrafast transport of holes from intercalated to neat region. The investigated samples 
could be classified into four distinct categories according to their microstructure and charge separation mechanism:

(1) One-phase, fully intercalated blend (1:1 pBTTT:PCBM blend processed at $35{ }^{\circ} \mathrm{C}$ ) - both electron and hole transfer occur on the $<100 \mathrm{fs}$ time scale, since no exciton diffusion is necessary within the single co-crystal phase. However, a majority of the electron-hole pairs recombine geminately within 200 ps.

(2) Two-phase microstructure-comprised of co-crystalline regions and PCBM clusters (1:4 pBTTT:PCBM blend processed at $35{ }^{\circ} \mathrm{C}$ ): electron transfer is prompt, while hole transfer can be prompt or delayed depending on whether excitons are generated in the intercalated or neat PCBM regions. Geminate charge recombination in the co-crystal phase is significantly reduced due to efficient migration of electrons toward fullerene clusters, favoring spatial separation of electron-hole pairs.

(3) Three-phase microstructure-with co-crystalline regions, PCBM clusters, and neat pBTTT domains (1:1 pBTTT:PCBM blend processed at room temperature, with different amounts of $\mathrm{Me} \mathrm{7}$, or with $1 \mathrm{~mol}$ equiv of $\mathrm{Me} \mathrm{12):} \mathrm{prompt} \mathrm{charge}$ separation occurs in the co-crystal phase and for excitons formed at the edge of neat domains, while exciton diffusion through PCBM or pBTTT regions limits the rate of charge separation (and leads to some loss of short-lived polymer excitons). Nevertheless, both neat PCBM and polymer domains contribute to the spatial separation of electron-hole pairs, reducing geminate recombination in intercalated regions. The holes promptly generated in the co-crystal phase migrate to neat $\mathrm{pBTTT}$ regions on the $<1$ ps time scale, showing that they are free to move immediately after their formation. This ultrafast hole transport is sustained by high local mobility (possibly along polymer chains extending from co-crystal to neat regions) and by an energy cascade driving the holes to the neat domains.

(4) Predominantly two-phase system-comprised of relatively phase-separated regions of neat PCBM and pBTTT (1:1 pBTTT:PCBM blend processed with $10 \mathrm{~mol}$ equiv of Me 12 or Me 14): charge separation becomes slow and multiphasic due to exciton diffusion, and there is significant loss to the ground state of excitons that cannot reach a charge separation site during their lifetime. Moreover, long-range fullerene ordering limits light harvesting by PCBM at $390 \mathrm{~nm}$, but remaining cocrystals can be selectively excited at this wavelength, followed by ultrafast transport of promptly generated holes to neat pBTTT regions.

Overall, the pBTTT:PCBM system has proven to be particularly suitable to answer many long-standing questions related to charge generation in polymer:fullerene blends. Not only because its microstructure can be precisely characterized and carefully manipulated, but also because the optoelectronic differences of the polymer found in neat or intercalated regions allow us to differentiate the electro-absorption according to the bulk heterojunction phase, giving us a unique tool to determine the location of the charges.

\section{ASSOCIATED CONTENT}

\section{S Supporting Information}

Experimental details, additional Figures as mentioned in the text, and electrostatic simulations. This material is available free of charge via the Internet at http://pubs.acs.org.

\section{AUTHOR INFORMATION}

\section{Corresponding Author}

*natalie.banerji@unifr.ch

\section{Funding}

M.S., M.C. and N.B. are funded by the Swiss National Science Foundation, through the Ambizione Fellowship (PZ00P2_136853) and the Stipend Professorship (PP00P2_150536). N.B. and M.C. are also supported by the University of Fribourg. J.D.J.R and J.E.M are supported by the NCCR-MUST from the Swiss National Science Foundation. N.S. is supported by a European Research Council (ERC) Starting Independent Researcher Fellowship under grant agreement No. 279587. E.B.D. is funded by a SABIC PostDoctoral Fellowship at King Abdullah University of Science and Technology.

\section{Notes}

The authors declare no competing financial interest.

\section{ACKNOWLEDGMENTS}

We thank Professor Eric Vauthey and Romain Letrun (University of Geneva) for putting at our disposition the fluorescence up-conversion setup and helping with the experiment. We like to also thank John R. Tumbleston and Harald Ade (Department of Physics, North Carolina State University), as well as Martin Pfannmöller and Rasmus R. Schröder (CellNetworks, BioQuant, Heidelberg University, Heidelberg, Germany) for their important contribution with respect to scanning transmission $\mathrm{X}$-ray- and transmission electron microscopy. We acknowledge Dr. Lee Richter and Dr. Dean Delongchamp from NIST for useful discussion concerning microstructure control using processing additives.

\section{REFERENCES}

(1) Green, M. A.; Emery, K.; Hishikawa, Y.; Warta, W.; Dunlop, E. D. Prog. Photovoltaics: Res. Appl. 2014, 22, 701.

(2) You, J.; Dou, L.; Yoshimura, K.; Kato, T.; Ohya, K.; Moriarty, T.; Emery, K.; Chen, C.-C.; Gao, J.; Li, G.; Yang, Y. Nat. Commun. 2013, 4, 1446.

(3) Thompson, B. C.; Fréchet, J. M. J. Angew. Chem., Int. Ed. 2008, $47,58$.

(4) Yu, G.; Gao, J.; Hummelen, J. C.; Wudl, F.; Heeger, A. J. Science 1995, 270, 1789.

(5) Brabec, C. J.; Sariciftci, N. S.; Hummelen, J. C. Adv. Funct. Mater. 2001, 11, 15.

(6) Beaujuge, P. M.; Fréchet, J. M. J. J. Am. Chem. Soc. 2011, 133, 20009.

(7) Lee, J. K.; Ma, W. L.; Brabec, C. J.; Yuen, J.; Moon, J. S.; Kim, J. Y.; Lee, K.; Bazan, G. C.; Heeger, A. J. J. Am. Chem. Soc. 2008, 130, 3619.

(8) Zhao, Y.; Xie, Z.; Qu, Y.; Geng, Y.; Wang, L. Appl. Phys. Lett. 2007, 90, 043504.

(9) Peet, J.; Kim, J. Y.; Coates, N. E.; Ma, W. L.; Moses, D.; Heeger, A. J.; Bazan, G. C. Nat. Mater. 2007, 6, 497.

(10) Hoppe, H.; Sariciftci, N. S. J. Mater. Chem. 2006, 16, 45.

(11) Ma, W.; Yang, C.; Gong, X.; Lee, K.; Heeger, A. J. Adv. Funct. Mater. 2005, 15, 1617.

(12) Shaheen, S. E.; Brabec, C. J.; Sariciftci, N. S.; Padinger, F.; Fromherz, T.; Hummelen, J. C. Appl. Phys. Lett. 2001, 78, 841.

(13) Huang, Y.; Kramer, E. J.; Heeger, A. J.; Bazan, G. C. Chem. Rev. 2014, 114, 7006.

(14) Liu, F.; Gu, Y.; Shen, X.; Ferdous, S.; Wang, H.-W.; Russell, T. P. Prog. Polym. Sci. 2013, 38, 1990.

(15) Westacott, P.; Tumbleston, J. R.; Shoaee, S.; Fearn, S.; Bannock, J. H.; Gilchrist, J. B.; Heutz, S.; de, M. J.; Heeney, M.; Ade, H.; 
Durrant, J.; McPhail, D. S.; Stingelin, N. Energy Environ. Sci. 2013, 6, 2756.

(16) Ruderer, M. A.; Meier, R.; Porcar, L.; Cubitt, R; MüllerBuschbaum, P. J. Phys. Chem. Lett. 2012, 3, 683.

(17) Pfannmöller, M.; Flügge, H.; Benner, G.; Wacker, I.; Kowalsky, W.; Schröder, R. R. Synth. Met. 2012, 161, 2526.

(18) Miller, N. C.; Cho, E.; Gysel, R.; Risko, C.; Coropceanu, V.; Miller, C. E.; Sweetnam, S.; Sellinger, A.; Heeney, M.; McCulloch, I.; Bredas, J.-L.; Toney, M. F.; McGehee, M. D. Adv. Energy Mater. 2012, 2, 1208.

(19) Collins, B. A.; Tumbleston, J. R.; Ade, H. J. Phys. Chem. Lett. 2011, 2, 3135 .

(20) Mayer, A. C.; Toney, M. F.; Scully, S. R.; Rivnay, J.; Brabec, C. J.; Scharber, M.; Koppe, M.; Heeney, M.; McCulloch, I.; McGehee, M. D. Adv. Funct. Mater. 2009, 19, 1173.

(21) Bartelt, J. A.; Beiley, Z. M.; Hoke, E. T.; Mateker, W. R.; Douglas, J. D.; Collins, B. A.; Tumbleston, J. R.; Graham, K. R.; Amassian, A.; Ade, H.; Fréchet, J. M. J.; Toney, M. F.; McGehee, M. D. Adv. Energy Mater. 2013, 3, 364.

(22) Jamieson, F. C.; Domingo, E. B.; McCarthy-Ward, T.; Heeney, M.; Stingelin, N.; Durrant, J. R. Chem. Sci. 2012, 3, 485.

(23) Kesava, S. V.; Fei, Z.; Rimshaw, A. D.; Wang, C.; Hexemer, A.; Asbury, J. B.; Heeney, M.; Gomez, E. D. Adv. Energy Mater. 2014, 4, 1400116.

(24) Buchaca-Domingo, E.; Ferguson, A. J.; Jamieson, F. C.; McCarthy-Ward, T.; Shoaee, S.; Tumbleston, J. R.; Reid, O. G.; Yu, L.; Madec, M. B.; Pfannmoller, M.; Hermerschmidt, F.; Schroder, R. R.; Watkins, S. E.; Kopidakis, N.; Portale, G.; Amassian, A.; Heeney, M.; Ade, H.; Rumbles, G.; Durrant, J. R.; Stingelin, N. Mater. Horiz. 2014, 1, 270.

(25) Dou, F.; Buchaca-Domingo, E.; Sakowicz, M.; Zhang, X.; Stingelin, N.; Silva, C. In SPIE: Physical Chemistry of Interfaces and Nanomaterials XII, San Diego, CA, 2013; Vol. 8811, p 88111J.

(26) Wang, T.; Pearson, A. J.; Lidzey, D. G. J. Mater. Chem. C 2013, 1,7266 .

(27) Kozub, D. R.; Vakhshouri, K.; Kesava, S. V.; Wang, C.; Hexemer, A.; Gomez, E. D. Chem. Commun. (Cambridge, U.K.) 2012, $48,5859$.

(28) Rance, W. L.; Ferguson, A. J.; McCarthy-Ward, T.; Heeney, M.; Ginley, D. S.; Olson, D. C.; Rumbles, G.; Kopidakis, N. ACS Nano 2011, 5, 5635.

(29) Savenije, T. J.; Grzegorczyk, W. J.; Heeney, M.; Tierney, S.; McCulloch, I.; Siebbeles, L. D. A. J. Phys. Chem. C 2010, 114, 15116.

(30) Baklar, M.; Wobkenberg, P. H.; Sparrowe, D.; Goncalves, M.; McCulloch, I.; Heeney, M.; Anthopoulos, T.; Stingelin, N. J. Mater. Chem. 2010, 20, 1927.

(31) Hamadani, B. H.; Gundlach, D. J.; McCulloch, I.; Heeney, M. Appl. Phys. Lett. 2007, 91, 243512.

(32) Mcculloch, I.; Heeney, M.; Bailey, C.; Genevicius, K.; I, M.; Shkunov, M.; Sparrowe, D.; Tierney, S.; Wagner, R.; Zhang, W. M.; Chabinyc, M. L.; Kline, R. J.; Mcgehee, M. D.; Toney, M. F. Nat. Mater. 2006, 5, 328.

(33) Paraecattil, A. A.; Banerji, N. J. Am. Chem. Soc. 2014, 136, 1472.

(34) Howard, I. A.; Mauer, R.; Meister, M.; Laquai, F. J. Am. Chem. Soc. 2010, 132, 14866.

(35) De, S.; Kesti, T.; Maiti, M.; Zhang, F.; Inganaes, O.; Yartsev, A.; Pascher, T.; Sundström, V. Chem. Phys. Lett. 2008, 350, 14.

(36) Barker, A. J.; Chen, K.; Hodgkiss, J. M. J. Am. Chem. Soc. 2014, $136,12018$.

(37) Burke, T. M.; McGehee, M. D. Adv. Mater. 2014, 26, 1923.

(38) Gélinas, S.; Rao, A.; Kumar, A.; Smith, S. L.; Chin, A. W.; Clark, J.; van der Poll, T. S.; Bazan, G. C.; Friend, R. H. Science 2014, 343, 512.

(39) Bittner, E. R.; Silva, C. Nat. Commun. 2014, 5, 3119.

(40) Howard, I. A.; Etzold, F.; Laquai, F.; Kemerink, M. Adv. Energy Mater. 2014, 4, 1301743.

(41) Grancini, G.; Maiuri, M.; Fazzi, D.; Petrozza, A.; Egelhaaf, H. J.; Brida, D.; Cerullo, G.; Lanzani, G. Nat. Mater. 2013, 12, 29.
(42) Jailaubekov, A. E.; Willard, A. P.; Tritsch, J. R.; Chan, W.-L.; Sai, N.; Gearba, R; Kaake, L. G.; Williams, K. J.; Leung, K.; Rossky, P. J.; Zhu, X. Y. Nat. Mater. 2013, 12, 66.

(43) Vithanage, D. A.; Devižis, A.; Abramavičius, V.; Infahsaeng, Y.; Abramavičius, D.; MacKenzie, R. C. I.; Keivanidis, P. E.; Yartsev, A.; Hertel, D.; Nelson, J.; Sundström, V.; Gulbinas, V. Nat. Commun. 2013, 4, 2334.

(44) Vandewal, K.; Albrecht, S.; Hoke, E. T.; Graham, K. R.; Widmer, J.; Douglas, J. D.; Schubert, M.; Mateker, W. R.; Bloking, J. T.; Burkhard, G. F.; Sellinger, A.; Fréchet, J. M. J.; Amassian, A.; Riede, M. K.; McGehee, M. D.; Neher, D.; Salleo, A. Nat. Mater. 2013, 13, 63.

(45) Dimitrov, S. D.; Bakulin, A. A.; Nielsen, C. B.; Schroeder, B. C.; Du, J.; Bronstein, H.; McCulloch, I.; Friend, R. H.; Durrant, J. R. J. Am. Chem. Soc. 2012, 134, 18189.

(46) Bakulin, A. A.; Rao, A.; Pavelyev, V. G.; van Loosdrecht, P. H. M.; Pshenichnikov, M. S.; Niedzialek, D.; Cornil, J.; Beljonne, D.; Friend, R. H. Science 2012, 335, 1340.

(47) Caruso, D.; Troisi, A. Proc. Natl. Acad. Sci. U.S.A. 2012, 109, 13498.

(48) Scarongella, M.; Paraecattil, A. A.; Buchaca-Domingo, E.; Douglas, J. D.; Beaupre, S.; McCarthy-Ward, T.; Heeney, M.; Moser, J. E.; Leclerc, M.; Fréchet, J. M. J.; Stingelin, N.; Banerji, N. J. Mater. Chem. A 2014, 2, 6218.

(49) Miller, N. C.; Cho, E.; Junk, M. J. N.; Gysel, R.; Risko, C.; Kim, D.; Sweetnam, S.; Miller, C. E.; Richter, L. J.; Kline, R. J.; Heeney, M.; McCulloch, I.; Amassian, A.; Acevedo-Feliz, D.; Knox, C.; Hansen, M. R; Dudenko, D.; Chmelka, B. F.; Toney, M. F.; Brédas, J.-L.; McGehee, M. D. Adv. Mater. 2012, 24, 6071.

(50) Spano, F. C.; Silva, C. Annu. Rev. Phys. Chem. 2014, 65, 477.

(51) Faist, M. A.; Keivanidis, P. E.; Foster, S.; Wöbkenberg, P. H.; Anthopoulos, T. D.; Bradley, D. D. C.; Durrant, J. R.; Nelson, J. J. Polym. Sci., Part B: Polym. Phys. 2011, 49, 45.

(52) Guilbert, A. A. Y.; Schmidt, M.; Bruno, A.; Yao, J.; King, S.; Tuladhar, S. M.; Kirchartz, T.; Alonso, M. I.; Goñi, A. R.; Stingelin, N.; Haque, S. A.; Campoy-Quiles, M.; Nelson, J. Adv. Funct. Mater. 2014, 6972.

(53) Banerii, N. J. Mater. Chem. C 2013, 1, 3052.

(54) Fürstenberg, A.; Julliard, M. D.; Deligeorgiev, T. G.; Gadjev, N. I.; Vasilev, A. A.; Vauthey, E. J. Am. Chem. Soc. 2006, 128, 7661.

(55) Jimenez, R.; Fleming, G. R.; Kumar, P. V.; Maroncelli, M. Nature 1994, 369, 471.

(56) Banerji, N.; Cowan, S.; Leclerc, M.; Vauthey, E.; Heeger, A. J. J. Am. Chem. Soc. 2010, 132, 17459.

(57) Banerji, N.; Cowan, S.; Vauthey, E.; Heeger, A. J. J. Phys. Chem. C 2011, 115, 9726.

(58) Wells, N. P.; Boudouris, B. W.; Hillmyer, M. A.; Blank, D. A. J. Phys. Chem. C 2007, 111, 15404.

(59) Meskers, S. C. J.; Hubner, J.; Oestreich, M.; Bassler, H. J. Phys. Chem. B 2001, 105, 9139.

(60) Reid, O. G.; Rumbles, G. J. Phys. Chem. Lett. 2013, 4, 2348.

(61) Guo, J.; Ohkita, H.; Benten, H.; Ito, S. J. Am. Chem. Soc. 2009, 131,16869 .

(62) Hwang, I.-W.; Kim, J. Y.; Cho, S.; Yuen, J.; Coates, N.; Lee, K.; Heeney, M.; McCulloch, I.; Moses, D.; Heeger, A. J. J. Phys. Chem. C 2008, 112, 7853.

(63) Hodgkiss, J. M.; Albert-Seifried, S.; Rao, A.; Barker, A. J.; Campbell, A. R.; Marsh, R. A.; Friend, R. H. Adv. Funct. Mater. 2012, 22, 1567.

(64) Lanzani, G. The Photophysics behind Photovoltaics and Photonics; Wiley-VCH Verlag \& Co: Weinheim, Germany, 2012.

(65) Cabanillas-Gonzalez, J.; Virgili, T.; Gambetta, A.; Lanzani, G.; Anthopoulos, T.; de Leeuw, D. Phys. Rev. Lett. 2006, 96, 106601.

(66) Devižis, A.; Hertel, D.; Meerholz, K.; Gulbinas, V.; Moser, J. E. Org. Electron. 2014, 15, 3729.

(67) Lee, M. J.; Gupta, D.; Zhao, N.; Heeney, M.; McCulloch, I.; Sirringhaus, H. Adv. Funct. Mater. 2011, 21, 932.

(68) Noriega, R.; Rivnay, J.; Vandewal, K.; Koch, F. P. V.; Stingelin, N.; Smith, P.; Toney, M. F.; Salleo, A. Nat. Mater. 2013, 12, 1038. 